\title{
ISOCAM mid-infrared detection of HR 10: A distant clone of Arp 220 at $z=1.44$
}

\author{
D. Elbaz ${ }^{1,2,3}$, H. Flores ${ }^{1}$, P. Chanial ${ }^{1}$, I. F. Mirabel ${ }^{1,4}$, D. Sanders ${ }^{5}$, P.-A. Duc ${ }^{1}$, \\ C. J. Cesarsky ${ }^{6}$, and H. Aussel ${ }^{5}$
}

1 CEA Saclay/DSM/DAPNIA/Service d'Astrophysique, Orme des Merisiers, 91191 Gif-sur-Yvette Cedex, France

2 Department of Physics, University of California, Santa Cruz, CA 95064, USA

3 Department of Astronomy \& Astrophysics, University of California, Santa Cruz, CA 95064, USA

4 Instituto de Astronomía y Física del Espacio, CONICET, 1428 Ciudad Universitaria, Buenos Aires, Argentina

${ }^{5}$ Institute for Astronomy, University of Hawaii, 2680 Woodlawn Drive, 96822 Honolulu, USA

${ }^{6}$ European Southern Observatory, Karl-Schwarzchild-Strasse 2, 85748 Garching bei München, Germany

Received 13 August 2001 / Accepted 30 October 2001

\begin{abstract}
We report the detection of the extremely red object (ERO), HR $10(I-K \sim 6.5, z=1.44)$, at 4.9 and $6.1 \mu \mathrm{m}$ (rest-frame) with ISOCAM, the mid-infrared (MIR) camera onboard the Infrared Space Observatory (ISO). HR 10 is the first ERO spectroscopically identified to be associated with an ultra-luminous IR galaxy (ULIG) detected in the radio, MIR and sub-millimeter. The rest-frame spectral energy distribution (SED) of HR 10 is amazingly similar to the one of Arp 220, scaled by a factor $3.8 \pm 1.3$. The corresponding $8-1000 \mu \mathrm{m}$ luminosity $\left(\sim 7 \times 10^{12} h_{70}^{-2} L_{\odot}\right)$ translates into a star formation rate of about $1200 h_{70}^{-2} M_{\odot} \mathrm{yr}^{-1}$ if $\mathrm{HR} 10$ is mostly powered by star formation. We address the key issue of the origin of the powerful luminosity of HR 10, i.e. starburst versus active galactic nucleus (AGN), by using the similarity with its closeby clone, Arp 220.
\end{abstract}

Key words. galaxies: evolution - infrared: galaxies

\section{Introduction}

HR 10 (or ERO J164502+4626.4, Dey et al. 1999), is the first and presently only Extremely Red Object (ERO, usually defined as galaxies with $I-K>4$ ) known to be associated with the class of ultra-luminous infrared galaxies (ULIGs, $\left.L_{\mathrm{IR}}=L(8-1000 \mu \mathrm{m}) \geq 10^{12} L_{\odot}\right)$. It was detected by $\mathrm{Hu}$ \& Ridgway (1994, hereafter HR) together with another ERO (HR 14 or ERO J164457+4626.0) in the field of the QSO PC $1643+4631 \mathrm{~A}(z=3.79)$. HR initially suggested that both galaxies with extreme colors $(I-K>6)$ could be distant ellipticals lying at $z \sim 2-3$. More generally, deep near IR (NIR) surveys indicate that EROs present the same clustering properties (Daddi et al. 2000; McCarthy et al. 2001) and surface brightness distribution (Moriondo et al. 2000) as elliptical galaxies. But dusty starbursts being potential progenitors of local ellipticals, they may also show similar clustering properties and some local examples, as NGC 7252 (Hibbard et al. 1994) or Arp 220 (Scoville et al. 2000), already show a de Vaucouleurs luminosity profile typical of ellipticals. High resolution NIR imagery and spectroscopy with the Keck

Send offprint requests to: D. Elbaz,

e-mail: delbaz@cea.fr telescopes (Graham \& Dey 1996) revealed that HR 10 was a moderately distant $(z=1.44)$ galaxy with an asymmetric morphology and $\mathrm{H} \alpha$ in emission. Another evidence against HR 10 being an early-type galaxy is its strong submillimeter luminosity as measured with SCUBA at the JCMT (Cimatti et al. 1998; Dey et al. 1999). The detection in HR 10 of a large CO luminosity, hence molecular hydrogen mass, was recently reported by Andreani et al. (2000) and presented as evidence favoring a star formation origin for the bulk of the IR luminosity rather than an AGN. However, a large gas mass may not only feed star formation but also gas accretion onto an AGN (see Papadopoulos et al. 2001). Another test for the presence of an AGN in a dusty galaxy is the warm over cold dust, i.e. mid IR (MIR, 3-40 $\mu \mathrm{m}$ ) over far IR (FIR, $40-300 \mu \mathrm{m}$ ), luminosity ratio as well as the shape of the MIR spectrum. We present the first detection of HR 10 in two MIR bands of ISOCAM onboard ISO corresponding to the rest-frame 3.3-6.1 and 4.9-7.4 $\mu \mathrm{m}$ wavelength ranges. A comparison to local galaxies SEDs is presented. We show that the SED of HR 10 is a scaled version of that of the closest ULIG, Arp 220. We discuss the origin of the luminosity of HR 10 on the basis of this similarity. 

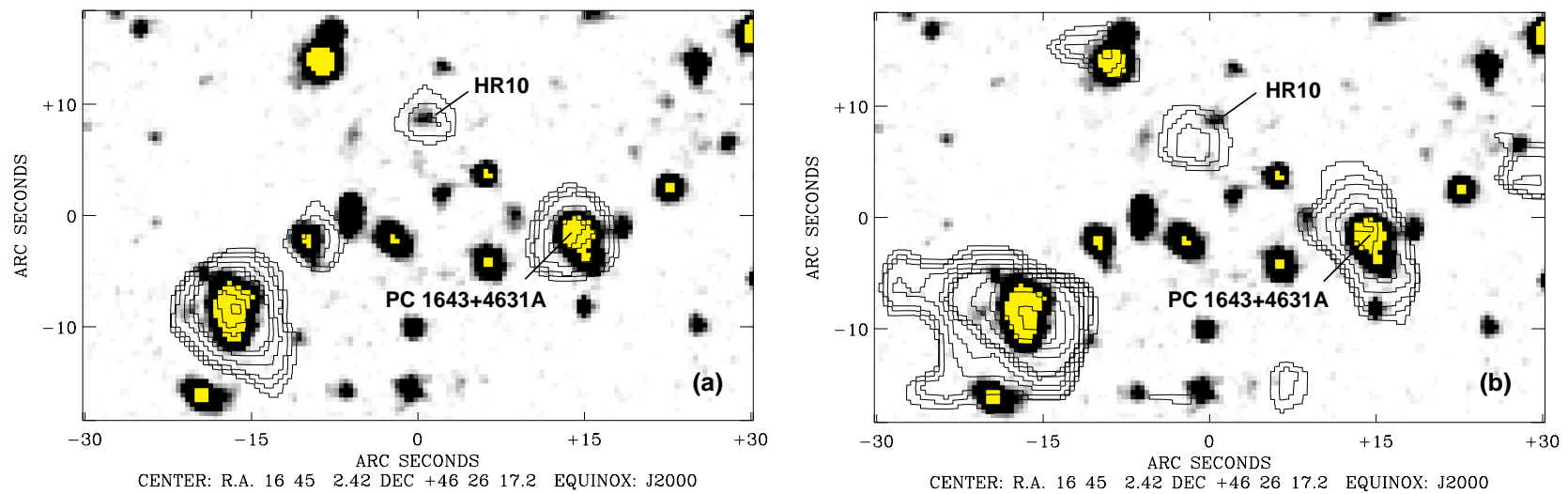

Fig. 1. MIR contours over $I$-band image (WHT archives); N: up, E: left. a) $15 \mu \mathrm{m}$ contours at 2.5 to $5-\sigma$, with a $0.5-\sigma$ step. b) $12 \mu \mathrm{m}$ contours at $S / N=1.6,1.9,2.2,2.8,3.5,4.1,6,9,11$.

Throughout this paper, we will assume $H_{0}=$ $70 \mathrm{~km} \mathrm{~s}^{-1} \mathrm{Mpc}^{-1}, \Omega_{\text {matter }}=0.3$ and $\Omega_{\Lambda}=0.7$. For this cosmology, the luminosity distance of $\operatorname{HR} 10(z=1.44)$ is $10373 \mathrm{Mpc}$.

\section{Observations}

Deep images of a field of $13^{\prime} \times 13^{\prime}$ centered on the QSO $\mathrm{PC} 1643+4631 \mathrm{~A}(z=3.79)$ were obtained with the ISOCAM broadband filters LW3 $(12-18 \mu \mathrm{m}$, centered at $15 \mu \mathrm{m})$ and LW10 $(8-15 \mu \mathrm{m}$, centered at $12 \mu \mathrm{m})$ for a total integration time of 16 and $13 \mathrm{~min}$ respectively. The $15 \mu \mathrm{m}$ image results from the coaddition of two overlapping mosaics slightly rotated one with respect to the other resulting in an improved spatial resolution ( $2^{\prime \prime}$ pixels). The $12 \mu \mathrm{m}$ image being made of one single mosaic of $6^{\prime \prime}$ pixels suffers from a lower spatial resolution. A given position of the sky was observed by 18 (20) different pixels at $15 \mu \mathrm{m}$ $(12 \mu \mathrm{m})$, resulting in a better flat-fielding and correction of cosmic ray impacts. The data reduction and source extraction was done with PRETI (Pattern REcognition Technique for ISOCAM data, Starck et al. 1999). The Gaussian noise (photon plus detector noise) in the $15 \mu \mathrm{m}$ $(12 \mu \mathrm{m})$ image is $58 \mu \mathrm{Jy}(31 \mu \mathrm{Jy})$. This field was also observed with ISOPHOT onboard ISO at 90 and $170 \mu \mathrm{m}$ (P.I. R.Ivison). The total exposure time per sky position is 11.5 and $6.6 \mathrm{~min}$ respectively. HR 10 is not detected with a 5 - $\sigma$ upper limit of $S_{\nu} \sim 200 \mathrm{mJy}$ in both bands.

In Fig. 1, the MIR contours are overlayed on the $I$-band image of a field containing both HR 10 and PC 1643+4631A. Both galaxies are detected in each MIR band with the flux densities given in Table 1. The uncertainty on the flux density related to the correction for the transient behavior of the detectors and to the pixel size relative to the point spread function is given in a second line in italics. While the $15 \mu \mathrm{m}$ contours are centered on the optical positions, the $12 \mu \mathrm{m}$ contours of HR 10 present an offset of $4^{\prime \prime}$. The astrometry was first calculated over the whole $13^{\prime}$ field using several stars from the US Naval Observatory Catalog which were detected in the MIR. A relative astrometric correction was then applyied to check the position of the MIR detection at the optical position of HR 10 by using a set of six objects included in a field of $2^{\prime} \times 2^{\prime}$ centered on HR 10 and including PC $1643+4631 \mathrm{~A}$. The probability of a chance association with an optical object with an $I$-band magnitude lower than $I$ within a distance $d$ was estimated using the following formula assuming a Poissonian distribution of sources: $P=1-\exp \left[-n(I) \pi d^{2}\right]$. The offset of $4^{\prime \prime}$ for the $12 \mu \mathrm{m}$ detection is attributed a probability of a chance association with the optical position of HR 10 of $20 \%$. This is not surprising because of the lower spatial resolution of the $12 \mu \mathrm{m}$ image ( $6^{\prime \prime}$ pixels). However the probability of a random association of the $12 \mu \mathrm{m}$ source within $4^{\prime \prime}$ of the $15 \mu \mathrm{m}$ detection of HR 10 is equal to $0.1 \%$ $\left(n[0.2 \mathrm{mJy}\right.$ at $\left.15 \mu \mathrm{m}]=0.8 \pm 0.1^{\prime-2}\right)$. In order to quantify the risk of detecting "ghost" sources produced by cosmic ray residuals in the MIR images, we performed MonteCarlo simulations by inserting fake sources in real datacubes. Down to a flux density limit of $S_{\nu} \sim 100 \mu \mathrm{Jy}$, we find that about 6 and $12 \%$ of the sources are false in the 15 and $12 \mu \mathrm{m}$ images respectively. However, we were able to reduce the fraction of ghost sources to zero in the $15 \mu \mathrm{m}$ image by requiring that a source be detected in both mosaics (before coaddition) at a lower detection level. Only above $0.3 \mathrm{mJy}$ do we reach the zero probability of a false detection in the $12 \mu \mathrm{m}$ image, but again the probability of a random association of a spurious $12 \mu \mathrm{m}$ source with the $15 \mu \mathrm{m}$ detection of HR 10 is negligible. The upper limits on HR $14(I-K=6.2$, see Table 1$)$ imply that it cannot be a ULIG unless it is more distant than $z \sim 1.8$. PC $1643+4631 \mathrm{~A}$ is one of the most distant sources detected in the MIR $(z=3.79$ corresponds to an age of the universe of only $1.6 \mathrm{Gyr}$ ) and belongs to the class of ULIGs, with rest-frame luminosities of: $\nu L_{\nu}[3.1 \pm 0.63 \mu \mathrm{m}]=(2.3 \pm 0.5) \times 10^{12} h_{70}^{-2} L_{\odot}$ and $\nu L_{\nu}[2.5 \pm 0.84 \mu \mathrm{m}]=(1.2 \pm 0.8) \times 10^{12} h_{70}^{-2} L_{\odot}$.

\section{Discussion: Nature of HR $\mathbf{1 0}$}

The least-square fit of the SED of HR 10 using the SED of Arp 220 (Fig. 2a) illustrates the amazing similarity of both 

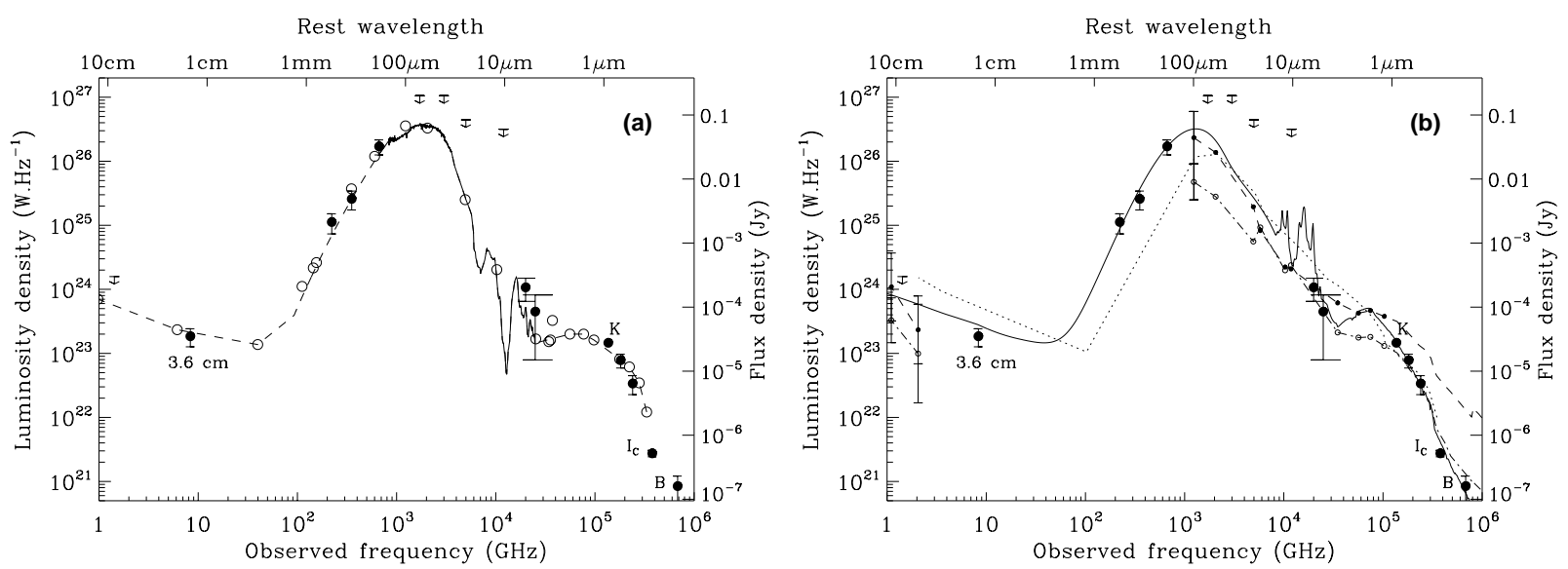

Fig. 2. Rest-frame SED of HR 10 (filled circles, Dey et al. 1999) including our MIR data. a) Comparison to the SED of Arp 220 normalized by a factor 3.8. Upper limits from IRAS $\left(\lambda_{\text {obs }}=25,60 \mu \mathrm{m}\right)$ and ISOPHOT $(90,170 \mu \mathrm{m})$. Plain line: combined spectra from ISO-LWS (43-185 $\mu \mathrm{m}$, Fischer et al. 1999) and ISOCAM-CVF (5-18 $\mu \mathrm{m}$, Charmandaris et al. 1999). Dashed line: interpolation between observed magnitudes: $U V$ (RC3), BI (Surace et al. 2000), RcJHKL (Kim 1995), IRAS (FSC), ISOPHOT (Klaas et al. 1997), sub-millimeter (Rigopoulou et al. 1996), radio (Anantharamaiah et al. 2000). b) Comparison to the mean SEDs of a "high reddening starburst" (dashed line, filled circles) and a Seyfert 2 (dash-dotted line, empty circles) from Schmitt et al. (1997), and to Mrk 231 (dotted line, Ivison et al. 1998).

Table 1. MIR flux densities at 15 and $12 \mu \mathrm{m}$, signal-to-noise ratios and probability of chance association.

\begin{tabular}{|l|rccrcc|}
\hline & $\begin{array}{r}S_{15 \mu \mathrm{m}} \\
(\mu \mathrm{Jy})\end{array}$ & $S / N$ & $\begin{array}{c}P \\
(\%)\end{array}$ & $\begin{array}{r}S_{12 \mu \mathrm{m}} \\
(\mu \mathrm{Jy})\end{array}$ & $S / N$ & $\begin{array}{r}P \\
(\%)\end{array}$ \\
\hline HR 10 & $203 \pm 58$ & 3.5 & 2.5 & $85 \pm 31$ & 2.2 & 20 \\
& $\begin{array}{r} \pm 2 \\
\text { PC 1643A }\end{array}$ & & & \pm 39 & & \\
& $330 \pm 58$ & 5.6 & 0.8 & $140 \pm 31$ & 3.6 & 2.0 \\
& \pm 12 & & & \pm 39 & & \\
HR 14 & $<290$ & 5 & - & $<135$ & 5 & - \\
\hline
\end{tabular}

spectra. The $8-1000 \mu \mathrm{m}$ luminosity of Arp 220 is $L_{\mathrm{IR}}=$ $1.8 \times 10^{12} h_{70}^{-2} L_{\odot}$, for a distance of $d=81.8 h_{70}^{-1} \mathrm{Mpc}$, corrected from the Virgo infall $\left(c z=5439+206 \mathrm{~km} \mathrm{~s}^{-1}\right)$. The resulting normalization factor of $3.8 \pm 1.3(1-\sigma)$ implies an IR luminosity of $L_{\mathrm{IR}}=(6.8 \pm 2.3) \times 10^{12} h_{70}^{-2} L_{\odot}$ for HR 10. If the dust was mostly heated by massive young stars, then this IR luminosity would translate into a star formation rate $(S F R)$ of about $(1170 \pm 396) h_{70}^{-2} M_{\odot} \mathrm{yr}^{-1}$, for a 10-100 Myr continuous burst, solar abundance and a Salpeter IMF $\left(S F R\left[M_{\odot} \mathrm{yr}^{-1}\right]=1.72 \times 10^{-10} L_{\mathrm{IR}}\right.$, Kennicutt 1998).

A consistent normalization factor also applies for the molecular gas mass of HR 10, estimated from its CO luminosity $\left(M_{\mathrm{H}_{2}}=1.2 \times 10^{11} M_{\odot}\right.$, Andreani et al. 2000), which is 2.9 times larger than in Arp $220\left(M_{\mathrm{H}_{2}}[\operatorname{Arp} 220]=\right.$ $4.1 \times 10^{10} M_{\odot}$, Scoville et al. 1997). If the molecular gas mass over dust mass ratio of HR 10 is the same as the one measured in the center of Arp 220, i.e. $M_{\text {gas }} / M_{\text {dust }} \sim 400$ (Scoville et al. 1997), then we obtain $M_{\text {dust }}[$ HR 10] $\sim 4 \times$ $10^{8} h_{70}^{-2} M_{\odot}$, in agreement with the one estimated by Dey et al. $\left(1999, \sim 7 \times 10^{8} h_{70}^{-2} M_{\odot}\right)$ using a black body fit to the FIR part of the SED with $T_{\text {dust }} \sim 40 \mathrm{~K}$.

The SED of Arp 220 is limited to wavelengths above the $U$-band $(3550 \AA)$ which corresponds to $8670 \AA$ in the observed frame of HR 10. In Fig. 2b, we have fitted the SED of HR 10 with the model STARDUST2 (Chanial et al., in preparation) from the observed $B$-band $(1800 \AA$ in the rest-frame) to the radio. If we assume that there is only one component responsible for both the optical and IR light in HR 10, then the best-fit is obtained for a visual extinction of $A_{V}=3 \mathrm{mag}( \pm 0.2 \mathrm{mag}$, extinction law $A(\lambda) / A_{V}$ from Calzetti et al. 2000), with an age of $0.2 \mathrm{Gyr}$ at the time of the observation and a characteristic timescale for the starburst of $\tau=95 \mathrm{Myr}$ $\left(S F R=M_{\text {gas }}(t) / \tau\right)$. The optical-UV part of the spectrum alone can be fitted with an $A_{V}=2-2.8$, an age of $2-4 \mathrm{Gyr}$ and $\tau=0.4-0.9 \mathrm{Gyr}$, resulting in $L_{\mathrm{IR}} \sim 8 \times 10^{10} h_{70}^{-2} L_{\odot}$ which does not fit the MIR and sub-millimeter part of the SED. However, detailed studies of local luminous IR galaxies have shown that the region from which the bulk of the IR luminosity arises can contribute very weakly to the optical light, e.g. the Antennae galaxies (Mirabel et al. 1998). In such a scenario, a second component with a larger $A_{V}$ would contribute dominantly to the IR regime and nearly not to the optical part. In both cases, we obtain an IR luminosity and $S F R$ within the error bars associated with the fit using Arp 220. The main difference between the model and Arp 220's SED is that the FIR over MIR luminosity ratio of Arp 220 is higher. The fit of Arp 220's SED requires an $A_{V}>30 \mathrm{mag}$ for the component responsible for the bulk of the IR light.

The key issue remains to determine whether the bulk of the luminosity of HR 10 is powered by star formation or accretion by a black hole. Two other EROs have been spectroscopically identified and detected in the MIR and radio (ISO J1324-2016, Pierre et al. 2001, and ERO J164023+4644, Smith et al. 2001). The upper limits established for their bolometric IR luminosities in the absence of a detection in the FIR or sub-millimeter are 
consistent with their belonging to the class of ULIGs too. NIR spectroscopy in both galaxies favor the presence of an AGN. In the case of HR 10, the width of the observed $\mathrm{H}_{\alpha}+[\mathrm{NII}]$ emission feature or the $[\mathrm{NII}] / \mathrm{H} \alpha$ ratio are too uncertain to be used as an indication of the presence of an AGN (Graham \& Dey 1996). The $\mathrm{H} \alpha$ line is strongly affected by dust extinction $\left(S F R[\mathrm{H} \alpha] \sim 80 M_{\odot} \mathrm{yr}^{-1}\right.$ only).

Most authors favor the starburst hypothesis as a dominant source of energy in Arp 220: its MIR spectrum up to $40 \mu \mathrm{m}$ shows no evidence for high ionization lines expected for AGNs (Sturm et al. 1996), its "IR excess" $\left(L_{\mathrm{IR}} / L[\mathrm{Ly} \alpha] \sim 24\right.$, Anantharamaiah et al. 2000) is much lower than for AGNs $(\sim 45-65)$ and typical of starburst galaxies $(\sim 12-45$, Genzel et al. 1998), its radio emission is produced by several compact sources (Smith et al. 1998), the ratio of aromatic features over MIR continuum (Genzel et al. 1998) and the slope of the MIR continuum (Laurent et al. 2000) are typical of starbursts. More recently, Haas et al. (2001) suggested that the MIR luminosity of Arp 220 could be underestimated because of dust extinction in the MIR and that after dereddening, its FIR over MIR luminosity ratio would be closer to the one for AGNs. But the dereddening factor varies by a factor five depending on the dust geometry assumed. Finally, the flat 2-10 keV hard X-ray spectrum of Arp 220 implies that in order to be mostly powered by an AGN, it would need to be Compton thick with a column density larger than $10^{25} \mathrm{~cm}^{-2}$ (Iwasawa et al. 2001).

In Fig. 2b, the SED of HR 10 is compared to the mean SEDs of a Seyfert 2 (Sy2) and of a starburst with high reddening (SBH), from Schmitt et al. (1997), normalized to the $15 \mu \mathrm{m}$ luminosity density of HR 10 . The 1- $\sigma$ error bars corresponding to the distribution of the $15 \mathrm{SBH}$ and $15 \mathrm{Sy} 2$ galaxies are shown in the radio and at $\lambda_{\text {rest }}=100 \mu \mathrm{m}$. Sub-millimeter data are missing in these SEDs but they should decrease in luminosity density above $100 \mu \mathrm{m}$ as in local galaxies, e.g. the Seyfert 1 Mrk $231\left(d=180.9 h_{70}^{-1} \mathrm{Mpc}, L_{\mathrm{IR}}=3.5 \times 10^{12} h_{70}^{-2} L_{\odot}\right.$, dotted line). Without MIR data, Mrk 231 was a candidate template for HR 10 (see Dey et al. 1999). Although not as red as HR 10, the SBH fits the MIR-FIR region of HR 10 while the FIR luminosity of the Sy2 is about ten times fainter.

Finally, even if the presence of a combination of an AGN and a starburst is still an option for both HR 10 and Arp 220, most studies favor a dominant contribution from star formation to their IR luminosities, implying that HR 10 presents the largest $S F R$ known at present. This study clearly shows the need for direct MIR and FIR/submillimiter observations of distant dusty galaxies to improve our understanding of a population of objects which plays a major role in galaxy evolution (Chary \& Elbaz 2001), hence emphasizes the importance of the next generation IR satellites to come, i.e. SIRTF, FIRST and NGST.

Acknowledgements. We wish to thank Guilaine Lagache for the reduction of the ISOPHOT images. DE wishes to thank the American Astronomical Society for their support through the Chretien International Research Grant, and Joel Primack \& David Koo for helping funding this research with the NASA grants NAG5-8218 and NAG5-3507.

\section{References}

Anantharamaiah, K. R., Viallefond, F., Mohan, N. R., Goss, W. M., \& Zhao, J. H. 2000, ApJ, 537, 613

Andreani, P., Cimatti, A., Loinard, L., \& Röttgering, H. 2000, A\&A, 354, L1

Calzetti, D., Armus, L., Bohlin, R. C., et al. 2000, ApJ, 533, 682

Charmandaris, V., Laurent, O., Mirabel, I. F., et al. 1999, Ap\&SS, 266, 99

Chary, R. R., \& Elbaz, D. 2001, ApJ, 556, 562

Cimatti, A., Andreani, P., Röttgering, H., \& Tilanus, R. 1998, Nature, 392, 895

Daddi, E., Cimatti, A., \& Renzini, A. 2000, A\&A, 362, L45

Dey, A., Graham, J. R., Ivison, R. J., et al. 1999, ApJ, 519, 610

Fischer, J., Luhman, M. L., \& Satyapal, S. 1999, Ap\&SS, 266, 91

Genzel, R., Lutz, D., Sturm, E., et al. 1998, ApJ, 498, 579

Graham, J., \& Dey, A. 1996, ApJ 471, 720

Haas, M., Klaas, U., Müller, S. A. H., Chini, R., \& Coulson, I. 2001, A\&A, 367, L9

Hibbard, J. E., Guhathakurta, P., van Gorkom, J. H., \& Schweizer, F. 1994, AJ, 107, 67

Hu, E. M., \& Ridgway, S. E. 1994, AJ, 107, 1303 (HR)

Ivison, R. J., Smail, I., Le Borgne, J.-F., et al. 1998, MNRAS, 298,583

Iwasawa, K., Matt, G., Guainazzi, M., \& Fabian, A. C. 2001, MNRAS, 326, 894

Kennicutt, R. C. Jr. 1998, ARA\&A, 36, 189

Kim, D.-C. 1995, Ph.D. Thesis, University of Hawaii

Klaas, U., Haas, M., Heinrichsen, I., \& Schulz, B. 1997, A\&A, $325, \mathrm{~L} 21$

Laurent, O., Mirabel, I. F., Charmandaris, V., et al. 2000, A\&A, 359, L887

McCarthy, P. J., Carlberg, R. G., Chen, H. W., et al. 2001, ApJ, 560, L131

Mirabel, I. F., Vigroux, L., Charmandaris, V., et al. 1998, A\&A, 333, L1

Moriondo, G., Cimatti, A., \& Daddi, E. 2000, A\&A, 364, 26

Papadopoulos, P., Ivison, R., Carilli, C., \& Lewis, G. 2001, Nature, 409, 58

Pierre, M., Lidman, C., Hunstead, R., et al. 2001, A\&A, 372, L45

Rigopoulou, D., Lawrence, A., \& Rowan-Robinson, M. 1996, MNRAS, 278, 1049

Scoville, N. Z., Yun, M. S., \& Bryant, P. M. 1997, ApJ, 484, 702

Scoville, N. Z., Evans, A. S., Thompson, R., et al. 2000, AJ, 119, 991

Schmitt, H. R., Kinney, A. L., Calzetti, D., \& Storchi Bergmann, T. 1997, AJ, 114, 592

Smith, H. E., Lonsdale, C. J., Lonsdale, C. J., \& Diamond, P. J. 1998, ApJ, 493, L17

Smith, G. P., Tommaso, T., Richard, E., et al. 2001, ApJ, accepted [astro-ph/0108039]

Starck, J.-L., Aussel, H., Elbaz, D., et al. 1999, A\&AS, 138, 365

Sturm, E., Lutz, D., Genzel, R., et al. 1996, A\&A, 315, L133

Surace, J. A., Sanders, D. B., \& Evans, A. S. 2000, ApJ, 529, 170 